\title{
Dynamique de l'expression silencieuse dans l'œuvre dramatique de Philippe Minyana
}

\author{
par \\ Dr. Alaaedin Baheidin Alaaedin*
}

Alaabahi364@gmail.com

\section{Resumé}

Nous proposons ici une étude sur l'écriture dramatique de l'écrivain Philippe Minyana. Nous pouvons dire que le but de notre travail est de savoir si Minyana voulu recréer le réel banal dans une pièce de théâtre classique avec un parole ordinaire qui arrive à toucher le public lorsqu'il essaie de restaurer cette banalité du quotidien avec une nouvelle construction. Notre étude sera consacrée sur le thème de l'expression silencieuse et son effet dans l'œuvre dramatique de Minyana, on va proposer les formes et les types différents de l'expression silencieuse utilisée par Minyana dans les trois pièces de notre corpus : «La Maison des morts », «Pièces » et «Retour», parce que le silence dans ces pièces représente un procédé linguistique qui donne une dimension poétique à son théâtre.

Mots clés: Dynamique - l'expression silencieuse - l'œuvre dramatique- Philippe Minyana

\section{Introduction}

Pour les auteurs contemporains, il paraît difficile de se poser ces questions: Qui raconte? Et comment raconter?, avec la fonction narrative qui a perdu l'efficacité qu'elle possédait autrefois, car: "La fonction narrative perd ses fonctions, le grand héros, les grands périls, les grands périples et le grand but." (Lyotard, 1979: 7).

* Professeur adjoint à la faculté des lettres- Université du Sud de la Vallée

(Dynamique de l'expression silencieuse ...) Dr. Alaaedin Baheidin Alaaedin. 
Parmi ces auteurs, Philippe Minyana s'impose comme un des plus grands auteurs contemporains français, c'est aussi l'un des plus joués et traduits. En 2010, il a reçu le grand prix du théâtre de l'Académie française pour l'ensemble de son œuvre:

"Minyana est un tritureur de langue, écrivant dans une rythmsation furieuse, ponctuation ouverte, phrases en équilibre entre parlé et écrit... c'est une parole grisante, et finalement jubilatoire..." (Azama, 2007: 96)

C'est pourquoi, il nous fallait offrir une nouvelle forme textuelle qui a la capacité de témoigner du monde d'aujourd'hui et en même temps qui peut répondre aux questions d'actualité: "Il en va de Minyana de ses confrères écrivains, créateurs de formes nouvelles, il invente le théâtre du XXIème siècle. Il le rêve, il le fabrique, il le modèle, il l'écrit... il travaille la matière de la langue..."(Corvin, 2000: 145). Quelques critiques dramatiques essaient d'approcher les nouvelles pièces parues à la fin du XXème siècle et le début de ce XXIème siècle, parmi lesquels, Patrice Pavis qui affirme: "Nous ne sommes pas encore occupés des "nouvelles écritures" après Beckett et Genet. Ces écritures, très novatrices, réclamant de tout nouveaux instruments d'analyse." (Pavis, 2007: 2).

Nous proposons ici une étude sur l'écriture dramatique de l'écrivain Philippe Minyana. Dans son théâtre, nous pouvons remarquer que son écriture est une écriture plus scénique que dramatique, la première paraît dominante dans son œuvre, et cela justifie la parole de Jean-Pierre Thibaudet: "On va voir un spectacle plus qu'une pièce." (Thibaudet, 2000: 10-11). Cet écrivain ne cherche pas à raconter une

(Dynamique de l'expression silencieuse ....) Dr. Alaaedin Baheidin Alaaedin. 
histoire, mais il avoue lui-même en affirmant à ce propos: "cela ne m'intéresse pas de raconter des histoires. Je préfère troubler." (Minyana, 2008: 115).

Donc, nous pouvons dire que le but de notre travail est de savoir si Minyana a voulu recréer le réel banal dans une pièce de théâtre classique avec une parole ordinaire qui arrive à toucher le public lorsqu'il essaie de restaurer cette banalité $\mathrm{du}$ quotidien avec une nouvelle construction théâtrale qui diffère totalement du format habituel d'une pièce de théâtre avec une langue poétique élaborée, car la: "question qui hante le théâtre, celle de son aptitude à représenter la réalité contemporaine, à mettre sur la scène le monde dans lequel nous vivons." (Dort, 1971: 7).

Notre étude sera consacrée essentiellement sur le thème de l'expression silencieuse et son effet dans l'œuvre dramatique de Minyana, on va proposer les formes et les types différents de l'expression silencieuse utilisés par Minyana dans les trois pièces de notre corpus: "La Maison des morts", "Pièces" et "Retour". parce que le silence représente dans les pièces de Minyana un procédé linguistique qui donne une dimension poétique à son théâtre: "Ecrire pour atteindre au silence, ramasser en quelques mots l'inoubliable du son, voilà sans doute ce qui porte vers la poésie." (Conort, 1988: 56).

(Dynamique de l'expression silencieuse ...) Dr. Alaaedin Baheidin Alaaedin. 


\section{I- L'écriture de Philippe Minyana}

"Il y a des écritures euphoriques, il y a des écritures tristes. Pourquoi? Je ne sais pas." (Minyana, Les cahiers de Prospero: décembre 1994: 45).

L'œuvre de Minyana, qui investit tous les champs de l'écrit, marque une étape importante dans la mise en question de la pièce de théâtre traditionnel qui a commencé au début du XXème siècle : "L'écriture contemporaine se préoccupe moins de la fiction, du personnage... de l'intrigue. Il y a "la parole". Il y a des "figures" qui prennent la parole. Et la parole emplit l'espace." (Minyana, 1999: 11). Philippe Minyana a découvert le théâtre par la mise en scène. Minyana a avoué l'influence de Vinaver sur lui, il a confirmé cet influence et surtout sur la langue utilisée dans son théâtre en disant que: "La langue, comme principe premier de l'écrit au théâtre, méticuleuse, "vraie", envisageable. Et puis, lui, l'homme, classe, très intelligent, émouvant." (Minyana, n. 2 1999: 26). Minyana a voulu concrétiser dans son théâtre ce que Vinaver a demandé à savoir un théâtre agissant: "par la mise en avant de l'oreille, oui, plus que de l'oil, qui doit rester accessoire." (Vinvar, 1982: 289). Il a déclaré d'après ses propres mots qu'il n'est pas seulement un auteur dramatique en disant que: "Je disais que je suis un écrivain de mots qui produisent du son (don du son ... le son fait son!), de la matière pour la scène." (Corvin, 2000: 145). C'est pourquoi, les œuvres différentes de Minyana se distinguent par une évolution d'écriture car: "Il y a beaucoup d'actions dans la façon dont la langue de Philippe Minyana est proférée..." (Fichet, 2000: 73). Ces œuvres sont issues de

(Dynamique de l'expression silencieuse ....) Dr. Alaaedin Baheidin Alaaedin. 
structures classiques, avec des protagonistes et une action définie, ces œuvres sont arrivées à une écriture proche du récit, dans laquelle, on trouve parfois la peinture, la photographie... Donc, il aborde le théâtre surtout par la pratique. Depuis trente ans, il a écrit une quarantaine de pièces. Parmi ses pièces: "Chambres", "Inventaires", "André", "Les Guerriers", "Où vas-tu Jérémié?", "Le Couloir", "Pièces", "La Maison des Morts" et "Voilà". Le thêâtre minyanesque s'articule autour des faits divers et banals comme une thématique centrale, selon la définition de Boussinot Roger, c'est le: "lieu commun, l'ordinaire, le médiocre, l'habituel et le fréquent." (Boussinot, 2001:256).

Dans son écriture, Minyana a cherché la brièveté: "Dire les mots, la pratique de l'écriture conduisent peu à peu le poète au silence, c'est dire que sa respiration décline jusqu'à son dernier souffle." (Pinget, 1985: 25).

L'œuvre de Minyana traite continuellement du théâtre, ce que l'on peut faire d'une écriture de théâtre pour la rendre mobile, loin du réalisme car: "Cette littérature et ce style retors restituent à l'œuvre sa consistance littéraire et rhétorique" (Pavis, 2016: 108). La question la plus présentée par les dramaturges et les critiques, c'est la puissance du théâtre à représenter la réalité contemporaine, à réactualiser le monde dans lequel nous vivons. Donc, c'est un théâtre de l'existence, c'est une chose primitive: "Le théâtre pour moi, ce sont des mots qui roulent, qui font du bruit." (Minyana, 25 février 1992). Dans ce thêâtre, la langue n'est ni quotidienne, ni réaliste, mais elle est un peu sophistiquée, c'est une langue singulière:

(Dynamique de l'expression silencieuse ...) Dr. Alaaedin Baheidin Alaaedin. 
"Minyana s'attaque de front nos chants, nos langues mouvementée, disjointes, nos errances dans les grammaires, les rythmes, surprenants de nos paroles vivantes... La trajectoire est simple, elle se situe au lieu du choix de la répétition, des mises en boucles..." (Huysmann, 2000: 95-96).

Donc, il s'agit de faire entendre la voix humaine dans tous ses mouvements, c'est pourquoi, la parole constitue la matière première de son théâtre. En bref, c'est un auteur qui fait parler le monde sur scène, Minyana a expliqué son point de vue en disant:

"Il m'a fallu créer ici un dialogue irréel, fait pourtant de mots courants, pour exprimer ce dont on ne parle pas... De telles conversations n'ont jamais lieu dans la réalité..." (Zande, Le Monde, 1967: 18).

\section{III- Théâtralité du silence}

Le théâtre de Minyana est un :"théâtre de l'aveu où la langue a une valeur thérapeutique." ( Corvin, 2000: 9-10).

Le principe de l'écriture et la forme adoptée dans chaque pièce diffèrent d'une pièce à l'autre en utilisant les mêmes procédés comme le recours au langage parlé, les pauses, disparition de ponctuation, ces procédés visent à peindre l'état de vie de ses personnages, Robert Cantarella affirme cela en disant:

"Le principe commun à toutes les pièces de Minyana est de questionner le monde tel qu'il $v a$, sans pour autant avoir un point de vue définitif sur l'écriture..." (Cantarella, 2000: 54).

(Dynamique de l'expression silencieuse ...) Dr. Alaaedin Baheidin Alaaedin. 
La représentation de la parole dans l'œuvre de Minyana a besoin d'une langue libérée et structurée, et notre travail est concentré sur l'expression silencieuse de Philippe Minyana. Le rapport qui se trouve dans son théâtre entre le silence et la parole est ambigu. Le silence se trouve dans l'œuvre de Minyana sous deux formes, soit explicite, soit implicite. Dans le premier genre, le texte nous montre clairement le silence par l'utilisation des didascalies du silence qui règlent les tours de paroles entre les personnages qui parlent comme: (Silence, Petite pause, Longue pause...etc.). Tandis que le second genre apparaît dans la parole des personnages qui: "ne sont pas des personnages mais des êtres qui portent des paroles... Et l'ensemble de ces êtres compose comme une vaste allégorie du monde." (Maragnant, 2000: 85).

Dans l'œuvre dramatique de Minyana, la parole devient visible par la voix des personnages, en plus, cette parole devient le principe de ce thêâtre et la source essentielle, d'où provient la poétique de la parole dans les pièces:

"Le poétique contraint le lecteur à s'arrêter sur la forme sensible du texte, matériau visible et sonore, chargé de correspondances avec le réel, qui se situe à la fois dans l'ordre du sens et dans l'ordre du sensible..." (Bourdieu, 1992: 159).

Les personnages de Minyana sont de simples gens, des voix de la foule, sans force et sans volonté, mais en même temps, ils portent une certaine importance:

(Dynamique de l'expression silencieuse ...) Dr. Alaaedin Baheidin Alaaedin. 
"Ils sont dans des situations extrêmes, ils portent la parole de l'aveu, ils représentent l'humanité..." (Huysmann, 2000: 98).

Egalement, ils sont les sujets de leur parole. Donc, nous pouvons préciser leurs caractères et leurs relations avec les autres en écoutant uniquement leurs paroles et savoir les traits langagiers qu'ils utilisent:

"Le personnage n'est au départ qu'un être de langage. Il existe seulement par et dans les mots." (Pruner, 2001: 81).

L'étude du silence dans l'œuvre dramatique de Minyana vise à connaître l'état des lieux et son rapport à la parole représentée.

En effet, la parole s'oppose au silence, le dernier arrête l'itinéraire du dialogue et du discours qui préparent les interlocuteurs à un temps d'émission de la parole. Cette parole est représentée sur scène pour qu'elle ait toutes les puissances possibles d'attirer l'attention du spectateur. Mais est-ce qu'on a besoin d'un temps de préparation pour le silence?

En répondant à cette question, nous remarquons tout d'abord que le théâtre de Minyana a donné la priorité à la parole par le biais du silence qui en fait une parole représentée. Le théâtre minyanesque s'intéresse par le silence qui vide la parole de son contenu pour faire introduire une autre voix. D'autre part, nous pouvons dire que ce silence s'anime dans la parole.

Dans le théâtre de Minyana, le manque de parole ne nécessite pas le silence comme (les bruits, les respirations, la musique...etc.), mais nous remarquons que dans la pause chez Minyana, on remarque cette volonté d'introduire dans

(Dynamique de l'expression silencieuse ...) Dr. Alaaedin Baheidin Alaaedin. 
les textes dramatiques un élément qui arrête la parole et fait parler le silence. Ainsi, le mouvement dans le texte dramatique est devenu un silence qui se transforme en parole, dès qu'il représente le manque de parole.

Dans notre travail, qui sera concentré sur l'étude du rapport de l'expression silencieuse à la parole représentée, nous voulons examiner ce rapport dans les pièces de notre corpus: "La Maison des morts", "Pièces" et "Retour".

\section{1- "La Maison des morts": l'expression à travers les didascalies}

"La Maison des morts" est un "texte nébuleux aux thématiques glacials et aux personnages fuyants, difficilement abordables d'un point de vue frontal." (Krol, 2012).

Il est à remarquer que "La Maison des morts" demande une certaine lecture vocale soit plus forte, soit moins forte avec l'aide de la typographie et surtout avec la présence des parenthèses qui nous force d'être attentifs en lisant la pièce. D'autre part, la parole dans la pièce suit les règles de l'écriture stylisée de Minyana, ce qui le distingue des autres écrivains et c'est la manière adoptée par lui tout au long de la pièce.

Selon Jean-Pierre Ryngaert, dans "La Maison des morts": "Rien ne s'est dit ni exprimé. Rien de logique ou de conséquent avec ce qui se suit. Aucune incidence sur rien, aucune préparation, aucun commentaire, aucune explication." (Ryngaert, 2008: 103). Les effets de l'expression silencieuse dans la pièce sont très clairs si bien qu'il est possible de constater que Minyana a voulu en faire

(Dynamique de l'expression silencieuse ...) Dr. Alaaedin Baheidin Alaaedin. 
les signes $\mathrm{du}$ ralentissement de la parole entre les interlocuteurs, c'est un travail très strict sur la langue:

"Le rythme de la pièce au stade de la création, pourrait être plus soutenu et irrégulier, ce qui placerait le spectateur dans une position encore plus inconfortable." (Krol, 2012).

La place qu'occupe l'expression silencieuse dans cette pièce nous paraît importante grâce à cette mise en parole que l'auteur montre tout au long de la pièce. C'est pourquoi, nous remarquons que la parole dans ce sens prend son souffle et ne recommence qu'après un temps d'arrêt entre les phrases comme dans cette scène:

"Elle nettoie avec précaution; la femme est allée s'asseoir dans son fauteuil, s'est perdue dans ses pensées. La Femme à la Natte attend, le chiffon à la main.

ET MES VALLAURIS/ MES VALLAURIS/ puis nettoie des vases/ ET MES RICHILIEU/ MES RICHELIEU/ puis nettoie des assiettes en porcelaine de Limoges/ ET MAINTENANT/ petite pause." (Minyana, La Maison des morts: 74).

Nous remarquons dans la scène ci-dessus que nous avons un silence explicite à la suite des déplacements des deux femmes, c'est pourquoi la parole suit les ordres de la Femme à la carapace, tandis que la femme à la Natte est parfaitement silencieuse, elle est forcée d'être dans un état de silence à cause de son travail de nettoyage.

Dans "La Maison des morts", la parole consiste sur les indications scéniques où nous ne pouvons pas comprendre et suivre le discours entre les interlocuteurs sans les lire. C'est

(Dynamique de l'expression silencieuse ...) Dr. Alaaedin Baheidin Alaaedin. 
pourquoi, nous trouvons que les didascalies s'avancent sur la parole du personnage pour expliquer ce qu'il veut dire.

Donc, les indications scéniques dans la pièce représentent les motivations pour les personnages qui parlent, elles sont plus importantes que les répliques elles-mêmes. Les indications économisent également la parole et facilitent la représentation et cela se manifeste dans l'exemple suivant:

"L'homme se lève, égaré, sort lentement du lit, traverse l'espace au ralenti, ouvre la porte, la referme; il est sorti. Cette séquence a pris un certain temps." (Minyana, La Maison des morts: 59)

Dans ce passage, nous remarquons que les indications scéniques délimitent l'état psychologique et physique de l'Homme aux Cannes, elle se trouvent à la place de la parole, elles décrivent les gestes de façon stricte. Grâce à ces indications scéniques, la représentation de cette séquences est palpable. Donc, nous pouvons dire que l'une des meilleures techniques de la représentation de Minyana dans cette pièce se trouve dans la valeur offerte aux gestes des personnages avant de présenter leur parole. Ces gestes règlent le rythme de la parole et ici apparaît le rôle de la mise en scène dans la pièce ce qui nous oblige à faire attention à l'emplacement des personnages et à leurs gestes qui précèdent leur parole.

Voilà pourquoi, nous trouvons que les indications scéniques sont pleines de pauses, de moments d'arrêt qui contiennent un silence clair:

"En réalité les didascalies délivrent des éléments d'information permettant un repérage des facteurs pertinents pour les tâches

(Dynamique de l'expression silencieuse ...) Dr. Alaaedin Baheidin Alaaedin. 
d'interprétation de message verbaux..."

(Gallepe, 226/1996: 136).

Minyana dans son article "L'écrit" paru dans Communication a affirmé qu'il a utilisé les didascalies fréquemment dans ses pièces en disant:

"J'inclus aussi beaucoup de didascalies dans mes pièces... Il y a cette envie d'accompagner la figure parlante dans son décor: savoir où elle parle, et ce qu'elle fait lorsqu'elle parle..." (Minyana, 2008: 116).

Donc, nous pouvons remarquer que la didascalie "Pause" signifie le silence. Cette indication "Pause" est fréquente dans l'œuvre dramatique de Minyana, et dorénavant dans son théâtre. De cette manière, Minyana voulait dire quelque chose puisque, la pause inclut un certain arrêt de la parole, c'est pourquoi, elle préfère sa mise en valeur, sa représentation sur scène, nous impose un temps lent lors de la lecture de la pièce; comme dans cet exemple:

"La Femme à la Natte:

Le geste

Petite pause

Avec la main

Petite pause

Vous l'avez faite

Petite pause

Comme ceci

Petite pause." (Minyana, La Maison des morts:

58)

Nous observons dans cet extrait la fréquence de la didascalie "Petite pause" dans la parole de la Femme à la Natte, cette didascalie impose un silence momentané qui met en évidence la parole de cette femme.

(Dynamique de l'expression silencieuse ...) Dr. Alaaedin Baheidin Alaaedin. 
Il est à remarquer également que la typographie du texte a joué un rôle important dans la mise en scène, et par suite, dans la façon d'être comprise de la part des comédiens. Ceux-ci se trouvent face à des séquences écrites selon le système de la typographie qui contient plusieurs didascalies détaillées réglant le mouvement de la parole comme l'affirme Michel Pruner lorsqu'il dit que le rôle de didascalie précise: "l'effet que l'auteur souhaite voir produit par le texte." (Pruner, 2005: 16).

Le silence se trouve entre ces séquences, car le texte de Minyana apparaît difficile à lire; et les acteurs doivent découper les séquences:

"L'homme se lève, égaré, sort lentement du lit, traverse l'espace au ralenti, ouvre la porte, la referme; il est sorti. Cette séquence a pris un certain temps. Apparaît un homme qui n'a qu'une jambe, qui n'a qu'un oeil, qui brandit un panneau où sont inscrits ces mots: J'ai honte mais j'ai faim." (Minyana, La Maison des morts: 59).

Dans cette scène, nous remarquons que le silence s'accorde avec les gestes de l'Homme, ce silence est exprimé par la didascalie. Cette didascalie ou cette indication scénique nous fournit une information qui explique la mauvaise situation de l'unijambiste.

D'autre part, nous trouvons le texte écrit en majuscule, ce qui donne l'impression d'un ton fort lors de la ponctuation de ce passage, comme dans l'exemple suivant:

"L'UNIJAMBISTE: PAS DE PROBLEME IL Y

A UN PURGATOIRE SÛR ET PAISIBLE OU

ON ATTEND QUE LA HONTE ET LE

(Dynamique de l'expression silencieuse ...) Dr. Alaaedin Baheidin Alaaedin. 


\section{REPENTIR LA PATIENCE ET LA BONNE}

CONDUITE VOUS TOMBE DESSUS."

(Minyana, La Maison des morts: 59).

La manière d'écrire cette pièce par Minyana nous fait découvrir l'importance du silence des acteurs qui accélèrent ou ralentissent le rythme de la parole dans la pièce.

Dans les sept mouvements de la pièce, nous remarquons que le silence et les blancs typographiques deviennent le signe le plus distinctif de la parole des personnages. Genviève Jolly affirme que le blanc typographique: "constitue même un discours propre, en surplombe du texte, sur ce que peut être le rythme scénique. S'il produit un effet visuel de discontinuité, le banc inscrit en outre la continuité d'une subjectivité." (Jolly, 2001: 113). Dans cette scène, la parole a perdu définitivement: "ce qui l'attire vers ses projets." (Ruzet, 2005: 77).

Une autre forme du silence se trouve dans "La Maison des morts", c'est dans l'échange des paroles où l'autre ne répond pas, et quand celui-ci commence à dire quelque chose, sa parole n'a aucune relation avec ce que vient de dire le premier, à l'inverse du théâtre classique, ou chacun a son tour de parole. Cette absence de communication dans les fragments exige l'activité des spectateurs pour rassembler tous les détails et comprendre le texte:

"... l'auteur reconstitue la marqueterie du réel, composant avec les bruits du monde et les paroles saisies au cru de la vie..." (Gwenola, 10 avril 2008).

Ainsi, le silence montre les moments de la parole effectuée où le personnage commence à lancer sa phrase selon les indications du texte, comme dans l'exemple suivant:

(Dynamique de l'expression silencieuse ...) Dr. Alaaedin Baheidin Alaaedin. 
"La Femme à la Natte:

BUVONS DES JUS DE FRUIT

La dame sort, la Femme à la Natte se lève, sert des jus de fruit.

J'aime boire des jus de fruit avec mes petites amies.

Elles boivent. Entrée de l'Homme aux Cannes. Il s'assied, soupire, pleurniche.

\section{L'Homme aux cannes:}

Et c'est le temps de pleurs des pleurs et des soupirs

Licy et Coco s'éloignent...

Petite pause..." (Minyana, La Maison des morts: 42-43)

Nous remarquons dans cette scène que la parole des personnages n'est pas en état d'échange, c'est-à-dire que personne ne répond à l'autre, les voix s'entrecroisent sur scène et cela nous donne un échange non communicable, il s'agit d'une scène dominée par la scène où la parole et le mouvement se mêlent.

Nous pouvons dire que le silence dans cette scène représente un élément indispensable de la parole. L'expression silencieuse chez Minyana est utilisée pour exprimer la perte de la volonté du personnage. Ce qui distingue le théâtre de Minyana dans cette pièce, c'est la confrontation entre la parole et le silence dans un face à face. La parole et le silence se présentent dans la pièce sans nier l'un l'autre. Pierre Larthomas a affirmé cette vision dans son livre "Le langage dramatique" en disant:

"Le déroulement d'une scène est faite de l'alliance de ces trois éléments, selon toutes les combinaisons possibles; parole- gestes-

(Dynamique de l'expression silencieuse ...) Dr. Alaaedin Baheidin Alaaedin. 
paroles; geste- silence- parole- silence, etc." (Larthomas, 1972: 260).

Une autre forme de silence représentée dans " $\underline{L a}$ Maison des morts": c'est le silence concrétisé par le personnage muet, qui est exempté de l'échange dialogique entre les interlocuteurs, mais sa présence complète la représentation que l'auteur veut sur la scène. Ce personnage est un témoin silencieux ou muet du drame qui se déroule sur la scène. Son rôle a plusieurs effets sur le drame. Premièrement, il donne une approbation au personnage qui parle et participe également dans la mise en évidence de la parole des autres personnages.

Ce personnage muet apparaît inutile d'une façon très claire à l'action, parce qu'il permet de valider la représentation. Son existence sur la scène est au même niveau que le décor et vise moins à achever un échange dialogique.

En bref, c'est un personnage exclu de parole, il est hors de l'action. En jouant un rôle sur la scène, il le joue en faisant certains gestes d'un façon mécanique comme n'importe quel accessoire sur la scène.

Ce silence cause la dépersonnalisation de ce personnage et l'empêche d'être autonome et dépend toujours de quelque chose qu'il représente ou de quelqu'un qui se trouve à côté de lui, cela s'accorde avec l'avis de Patrice Pavis, dans son "Dictionnaire du théâtre" qui nous explique que le vrai silence, c'est le silence métaphysique:

"le seul silence qui ne se réduit pas aisément à une parole à voix basse ne semble pas avoir d'autre cause qu'une impossible congénital à communiquer... ou une condamnation à jouer

(Dynamique de l'expression silencieuse ...) Dr. Alaaedin Baheidin Alaaedin. 
avec les mots sans pouvoir les relier aux choses sur un autre mode que ludique." (Pavis, 2002:

327).

C'est pourquoi, nous remarquons qu'il y a une perte de relations, c'est-à-dire, perte: "des liens entre la créature et son auteur... entre l'ancien pôle du caractère et les paroles prononcées et la situation..." (Ryngaert, 2008: 104)

Egalement, nous trouvons cet homme muet concrétisé dans le cas de l'Homme malade qui reste dans son lit, tandis que sa femme et la voisine échangent un dialogue avec la Femme à la Natte comme dans l'extrait suivant qui explique clairement ce genre de ce personnage muet:

"L'Homme malade:

Ma chatte

Fait signe à la femme de s'approcher; elle s'approche; il parle à voix basse. Ensuite, ils se regardent. Elle, avec une grande intensité. Lui, regard chien battu. Il gémit, tourne la tête, coté cloison. Lui fait à nouveau signe d'approcher, lui parle bas. Puis regards entre eux. Même scénario que précédemment." (Minyana, La Maison des morts: 56-57).

Comme nous avons déjà vu que cet homme malade est privé du dialogue, il représente en ce cas une forme parfaite de silence. Cette expression silencieuse ne forme pas une partie du dialogue, mais elle est soumise à ce dialogue malgré qu'elle ne participe même pas à produire ce dialogue. Son existence muette le rend une figure ambiguë et inutile à l'action, son existence ne diffère pas trop avec la présence d'un décor abstrait sur la scène, c'est pourquoi, ce personnage muet n'a pas d'influence sur l'action et la parole qu'on trouve dans l'échange des autres interlocuteurs. Sa privation du

(Dynamique de l'expression silencieuse ....) Dr. Alaaedin Baheidin Alaaedin. 
dialogue fait de lui un récepteur passif de la parole des autres interlocuteurs.

En somme, dans "La Maison des morts", l'expression silencieuse est représentée dans les indications scéniques dans les sept mouvements de la pièce, et donc, c'est un silence créé par l'auteur qui vise à confirmer les moments de prise de parole des personnages dans les lieux clos où ils n'arrivent pas à parler l'un à l'autre. Cette difficulté apparaît avec les autres personnages, mais également avec lui-même, c'est ainsi que sa parole exige un silence.

\section{2- "Pièces": L'expression silencieuse à travers le}

\section{personnage neutre}

Dans l'œuvre dramatique de Minyana, "Pièces" représente la pièce la plus silencieuse. La pièce est formée de neuf tableaux et dans chaque tableaux, il $\mathrm{y}$ a des parties où Minyana nous présente des gens très banals qui vivent en marge de la société. Minyana a essayé de dépeindre dans cette pièce la réalité telle quelle en nous offrant son personnage essentiel qui concrétise la difficulté de l'existence. Mais, il paraît très important de savoir la dynamique de l'expression silencieuse dans une pièce où les indications scéniques représentent l'axe essentiel du texte, car cette indication scénique n'est pas: "un texte d'appui, car elle ne vise ni à compléter, ni à préciser, mais plutôt à déconstruire un référent qu'elle est censée construire et à s'aborder dans ces cas extrêmes." (Calas, 2007: 71).

Les indications scéniques dans "Pièces" ont pour rôle de ralentir les mouvements des interlocuteurs sans resserrer l'action comme dans l'extrait suivant:

(Dynamique de l'expression silencieuse ...) Dr. Alaaedin Baheidin Alaaedin. 
"Une pièce. Quelques chaises. Un paravent. Deux petits rideaux qui ont la fonction de porte. Aux murs, expo photos. Portraits d'hommes, visages émaciés. Devant le mur, Femme assise (à l'extérieur). Femme presse un bouton. Bruit caractéristique d'une porte qui s'ouvre. Petite pause..." (Minyana, Pièces: 107).

Dans ce passage, nous remarquons que la parole est rare, parce que les gestes des deux interlocuteurs se contentent de donner quelques mots de temps en temps. Le temps d'attente a son effet, il crée un silence clair et la parole est guidée par les indications scéniques.

Comme nous voyons que dans "Pièces", les indications scéniques règlent le discours des personnages. Elle forment une partie inséparable du texte en jouant le rôle de guide qui détermine les tours de la parole et de discours pour les lecteurs. Egalement, elles sont très importantes pour comprendre l'action en expliquant les gestes des interlocuteurs.

Ce silence suggéré par les indications scéniques est fréquent dans les neuf tableaux de "Pièces". C'est le silence d'un personnage neutre dans une scène où les deux interlocuteurs parlent, chacun à son tour, l'un parle et l'autre, puis se tait et vice versa.

Cette parole échangée entre les deux personnages a contribué à créer ce personnage neutre qui a concrétisé le silence dans la pièce. Cela se manifeste clairement dans la scène introductive quand Tac écoute les autres, ce personnage souffre d'un état de déséquilibre avec lui-même en affrontant une sorte d'inquisition par les autres

(Dynamique de l'expression silencieuse ...) Dr. Alaaedin Baheidin Alaaedin. 
personnages. Il reste silencieux en assistant à cette perquisition d'objets faite par les autres devant lui:

"Chez Tac. Pièce vide. (Carreau cassé).

Papier peint et sur le mur portrait photographique d'Odette Tac. Tac, assis, oil écarquillé. La gardienne ouvre et ferme la porte. Le charcutier, portant paquet, est entré, parle (à voix basse) à l'oreille de la Gardienne. Gardienne dit à Tac: "C'est monsieur Bas qui rend la literie." Tac dit: "Merci". Autre coup de sonnette (forté). Gardienne ouvre et ferme la porte..." (Minyana, Pièces: 84 ).

Après avoir lu l'extrait ci-dessus, nous remarquons que Tac ne parle pas, c'est un personnage indifférent ou neutre, il ne s'intéresse pas à ce qui se passe devant lui ou ce qui se dit de la part des autres personnages. Son langage devient de plus en plus neutre en se contentant de donner quelques courtes répliques devant les autres personnages. Michel Foucault a expliqué cette neutralité dans le langage en disant:

"il se retire du milieu des êtres pour entrer dans son âge de la transparence et de neutralité." (Foucault, 1984: 70).

D'autre part, nous pouvons rencontrer de longues tirades accompagnées par un silence au cours de la tirade. Cela se manifeste évidement dans une scène intitulée "Les deux cousins". Dans cette scène, nous remarquons que la Bonne parle de sa vie privée, sa parole est coupée par des périodes de silence si le texte n'est pas articulé. Ces moments d'arrêt nous permettent d'avoir un recours aux marques dans le texte qui ralentissent le ton de la parole du personnage:

"La Bonne dit: Mon mari qui est monté sur le toit pour remplacer des tuiles après une tempête

(Dynamique de l'expression silencieuse ...) Dr. Alaaedin Baheidin Alaaedin. 
est tombé il est mort comme je n'avais pas de profession ni mes enfants non plus comment payer les traites les organismes sociaux nous ont logés (c'était loin de l'agglomération un exbâtiment humide et puis le temps a ralenti c'était après midi je me croyais le soir..." (Minyana, Pièces: 117).

Dans cette longue tirade, nous remarquons qu'il y a une présence de pauses au cours du discours, ce qui nous mène au silence. Si l'auteur a voulu ralentir la tirade, cela exige de lui de faire attention au mouvement intérieur du texte. Il doit être également sensible à la dynamique du verbe qui porte le sens du silence. Ainsi, la tirade porte une gêne moins à cause de ce qu'elle représente qu'à cause du silence qu'elle implique chez l'interlocuteurs.

Nous remarquons, la présence des parenthèses dans "Pièces". Cela aide les lecteurs à lire le texte d'un ton et d'une manière différents, ainsi que ces mots mis entre parenthèses permettent aux acteurs de les lire de façon différente.

\section{3- "Retour": le silence fait partie de la parole}

Dans la troisième pièce de notre travail "Retour", le silence se manifeste clairement dans la parole quotidienne dans un récit qui provoque la mémoire de la femme, c'est pourquoi, nous trouvons ici le silence que nous pouvons considérer comme un résultat d'un ralentissement de la parole:

"le théâtre conserve une capacité d'intervention
sur le réel à travers le jeu de ses figures, par sa
manière inimitable... de mobiliser les capacités

(Dynamique de l'expression silencieuse ...) Dr. Alaaedin Baheidin Alaaedin. 
imaginaires et corporelles des individus.

(Abirashed, 2006: 16-17).

Il est à remarquer dans "Retour", que le silence a occupé une place importante en faisant partie de la parole. Le thème du silence dans cette pièce nous paraît problématique, il provient du vide qu'on peut remarquer entre les répliques et qui donne un rythme au contenu:

"les mots petit à petit se vident de sens et deviennent des sons orchestrés." (Demey, 22 octobre 2007).

Donc, l'expression silencieuse dans l'œuvre dramatique minyanesque apparaît importante dans la mesure où elle forme un fond sur lequel s'étend la parole. Séverine Ruzet affirme ce point de vue en disant:

"Le silence s'impose comme signifié, mais également comme signifiant. Quand les silences ne s'enchaînent plus, quand elles manquent à atteindre leurs possibles destinataires, la parole ne parvient pas à combler les distances, et des trous noirs viennent graver le dialogue." (Ruzet, 33/2005: 78).

Ce silence qui n'est accompagné par aucune action acquière plus d'importance. Il représente le temps qui passe, c'est le temps que l'auteur crée entre les séquences ou entre un mot et le mot qui suit. Ce silence est parfois visé par un signe typographique.

Dans la pièce "Retour", cette importance accordée par Minyana au sujet du silence a donné en effet la valeur à la parole des personnages dans le récit et présente des arrêts qui attirent l'attention des personnages sur ce qui se passera, car les personnages de cette pièce sont des:

(Dynamique de l'expression silencieuse ...) Dr. Alaaedin Baheidin Alaaedin. 
"mécaniques, répétitifs, débitent leurs répliques et le pointent du doigt. Ils tapotent. Se grattent. S'impatientent. Ponctuant leurs propos de gestes démonstratifs." (Demey, 22 octobre 2007).

Egalement, dans "Retour", le silence crée des espaces entre les séquences et forme une opposition de la parole, comme dans l'exemple suivant:

"Et je revois (en pensée) José qui fait du synthé

Et je revois (en pensée) Adeline qui tient une cantine

Et je revois (en pensée) Luc qui joue du trombone

Et je revois (en pensée) Valérine qui fait de la photo

Et je revois (en pensée) Denise qui vend des boutons, etc." (Minyana, Pièces: 88 ).

A cause de l'absence totale de didascalies, le silence dans la pièce est évident au niveau des phrases, ainsi qu'entre les séquences dans le récit qui sont séparées par des espaces. Egalement, nous observons la répétition de plusieurs phrases comme des refrains dans le texte. C'est pourquoi, le silence provient de cette reprise des phrases où l'acteur répète ces phrases du texte, comme dans l'exemple:

"Revenant dans mon pays je me dis ah c'est mon pays et le temps s'est mis à ralentir et tout paraît retrouver sa fonction naturelle je traverse les paysages ainsi s'accomplit le périple." (Minyana, retour: 75).

Cette phrase représente un leitmotiv qui se répète dans certains endroits dans le texte. Cette phrase répétée crée un changement vocal et arrête la parole de ce qui la précède.

(Dynamique de l'expression silencieuse ...) Dr. Alaaedin Baheidin Alaaedin. 
Donc, le silence, comme nous remarquons n'est pas clair dans ce cas, mais il est inclus dans le texte.

D'autre part, nous observons dans ce passage l'absence totale de la ponctuation, cela nous aide à lire le texte de plusieurs façons. Cette absence donne au texte une vitalité du verbe et du mot à l'intérieur de chaque phrase, elle permet également à donner une liberté de lecture du texte et qui aboutit à la fin à une représentation plus facile de la parole sur la scène. Donc, on peut dire que Minyana a adopté cette absence de ponctuation comme un procédé pour nous faire parvenir le contenu du dialogue, Michel Corvin a déclaré son point de vue en disant:

"l'absence de ponctuation écrase le temps, elle égalise les effets, elle révèle et dénonce les arrières pensées." (Corvin, 2000: 21).

De son côté, Minyana nous a donné la raison principale de cette absence de ponctuation dans ses pièces et l'effet de cette absence en disant:

"Il n'y a pas de ponctuation dans les textes, il y a donc une sorte de vertige de la parole, une abondance de la parole..." (Minyana, 25 février 1992).

D'autre part, l'écriture de Philippe Minyanna dans "Retour", est fondée sur le quotidien, cela se manifeste évidemment dans la parole des personnages dès les premières lignes de l'œuvre, voilà pourquoi, nous retrouvons plusieurs fragments dans la pièce provenant du langage quotidien comme dans l'exemple suivant:

"et le boulot ça va le boulot et la santé ça va la santé." (Minyana, Retour: 79).

(Dynamique de l'expression silencieuse ...) Dr. Alaaedin Baheidin Alaaedin. 
De cette manière, nous pouvons dire que Philippe Minyana nous a présenté le réel avec clarté.

Nous observons également dans "Retour" que Minyana a utilisé une technique habituelle pour lui, c'est l'utilisation des parenthèses. En utilisant ce procédé technique, Minyana a crée des effets et un changement vocal.

En bref, il est important de lire le texte à haute voix afin de préciser et de savoir les moments de la parole et les moments du silence dans lesquels, les personnages font une promenade silencieuse dans l'espace en créant un temps entre les séquences de la parole.

\section{Conclusion}

Dans l'œuvre dramatique de Minyana, le silence représente un élément indispensable dans la parole théâtrale chez lui. Nous avons observé également à travers les trois pièces de notre corpus: "La Maison des morts", "Pièces" et "Retour", que le silence est abordé dans chaque pièce d'une façon différente. Cela nous aide à mieux comprendre la représentation de la parole dans le théâtre minyanesque en concentrant sur les trois pièces étudiées.

Dans l'œuvre dramatique de Minyana, celui-ci a appliqué plusieurs procédés de représentation le silence selon la forme dramatique de chaque pièce, il n'y a pas une manière unique pour exprimer le silence dans toute l'œuvre, mais il y a certains éléments qui s'entrecroisent et qui forment une sorte d'unité dramatique.

La diversité des formes de l'expression silencieuse dans l'œuvre de Minyana nous a permis de remarquer

(Dynamique de l'expression silencieuse ...) Dr. Alaaedin Baheidin Alaaedin. 
l'écriture du silence dans l'œuvre, ainsi que le traitement du silence dans les pièces étudiées dans notre travail.

L'attente dans le théâtre de Minyana est fréquente, cette attente signifie le silence. La parole vient fortifier sa valeur et son effet dans les situations dramatiques entre les interlocuteurs.

Dans notre travail, nous avons mis la lumière sur les formes diverses $\mathrm{du}$ silence dans les trois pièce. Premièrement, le silence dans le texte, où il n'y a pas de discours qui se concrétise dans le refus de parler. Ce genre du silence se manifeste clairement à travers les personnages qui refusent de participer dans la conversation comme dans le cas de Tac dans "Pièces", et également dans le cas du personnage muet dans "La Maison des morts".

Deuxièmement, c'est le silence des indications scéniques qui couvrent la pièce comme les points de suspension (petite pause, temps, silence...). Cette forme de l'expression silencieuse paraît dans "La Maison des morts" et dans "Pièces".

La dernière forme de l'expression silencieuse représente le sommet de l'écriture silencieuse, dans ce genre de silence, la langue, c'est elle qui crée le silence. Nous pouvons toucher ce genre dans les tirades où le silence s'intègre dans la parole, et l'arrêt de cette parole se passe au sein même du discours. Cet arrêt peut sortir à la suite d'un changement de ton, c'est le cas de la parole mise en italique ou la parole entre parenthèse.

Donc, l'écriture du silence apparaît au cœur du texte, elle paraît à l'inverse de l'écriture des indications scéniques, puisque le silence n'est pas palpable. Cette forme de silence

(Dynamique de l'expression silencieuse ...) Dr. Alaaedin Baheidin Alaaedin. 
apparaît dans le récit, il surgit quand il y a un besoin du silence entre les séquences de la parole. Nous pouvons trouver ce type de silence dans "La Maison des morts" où les personnages utilisaient une parole, dans laquelle, ils apparaissent incapables d'aller au bout de la phrase.

Finalement, la représentation de la parole à travers de l'expression silencieuse dans les pièce de Philippe Minyana est très évidente à travers les techniques différentes utilisées par Minyana. C'est ce que nous avons essayé d'étudier dans notre travail. 


\section{Bibliographie}

I- Le Corpus

1- MINYANA, Philippe, La Maison des morts, Paris, Editions Théâtrales, 1996.

2- MINYANA, Philippe, Habitation, Pièces, Paris, Edition Théâtrales, 2001.

3- MINYANA, Philippe, Retour, Paris, Edition Théâtrales, 2004.

\section{II- Ouvrages Généraux}

1- BOURDIEU, Pierre, Les règles de l'art, Paris, Seuil, 1992.

2- BOUSSINOT, Roger, Dictionnaire Bordas des synonymes, analogies, antonymes, Paris, Larousse, 2001.

3- CALAS, Frédéric, Le didascalique à l'épreuve de sa représentation, Presses Universitaires de Bordeaux, 2007.

4- DORT, Bernard, Théâtre réel, Paris, Seuil, 1971.

5- FOUCAULT, Michel, Les mots et les choses, Paris, Gallimard, 1984.

6- LARTHOMAS, Pierre, Le langage dramatique, Paris, Armand Colin, 1972.

7- LIOURE, Michel, Lire le théâtre moderne de Claudel à Ionesco, Paris, Nathan, 2002.

8- LYOTAED, Jean François, La condition postmoderne, Paris, ed. de Minuit, 1979.

9- PAVIS, Patrice, Le Dictionnaire du théâtre, Paris, Armand Colin, 2002.

(Dynamique de l'expression silencieuse ...) Dr. Alaaedin Baheidin Alaaedin. 
$10-$ , Le théâtre contemporain: Analyse des textes de Sarraute à Vinaver, Paris, Armand Colin, 2007. 11 L'analyse des textes dramatiques de

Sarraute à Pommerat, Paris, Armand Colin, 2016.

12- PINGET, Robert, Charrue, Paris, Minuit, 1985.

13- PRUNER, Michel, L'analyse du texte de théâtre, Paris, Armand Colin, 2005

14-THIBAUDAT, Jean-Pierre, Théâtre français contemporain, Paris, Ministère des affaires étrangères, 2000. 15- VINAVER, Michel, Ecrits sur le thêâtre, Paris, Lausanne, L'Aire, 1982.

\section{$\underline{\text { II- Ouvrages consacrés à Minyana et son oeuvre }}$}

1- CANTARELLA, Robert, Anne-Marie suivie de Les Ecritures de Philippe Minyana, in Théâtre Ouvert, Paris, 2000.

2- CORVIN, Michel, Philippe Minyana ou la parole visible, Paris, ed. Théâtrales, 2000.

3- HUYSMANN, Christophe, "Philippe Minyana, un artiste dans la langue", in "Philippe Minyana, ou la parole visible", Paris, éditions Théâtrales, 2000.

4- MARAGNANT, Frédéric, "Projet d'écriturel pratique de l'acxteur/technique sur la scène", in "Philippe Minyana ou la parole visible", Paris, ed. Théâtrales, 2000.

(Dynamique de l'expression silencieuse ...) Dr. Alaaedin Baheidin Alaaedin. 


\section{III- Articles de revues}

1- ABIRASHED, Robert, "La situation faite au théâtre d'aujourd'hui", Dialogue- débat au Centre National du Livre, publié dans La Terrasse, n. 41, Paris, 2006.

2- AZAMA, Michel, "De Godot à Zucco, une anthologie", in Thêâtre Public, N. 184, 2007.

3-CONORT, Benoît, "Pour une poétique du contradictoire", in Les Silences, Revue Recueil, n 8, 1988.

4- DEMEY, Eric, "Une suite sur le retour d'un homme", in Les Trois Coups, 13 février 2012.

5- FICHET, Roland, dans "Anne-Marie suivie de Les Ecritures de Philippe Minyana", Paris, Editions Théâtrales, 2000.

6- GALLEPE, Thierry, "Les incidences des didascalies dans la mise en scène de la parole", in Cahiers de praxématique, Montpellier, 26/ 1996, pp. 135-176.

7- GWENOLA, David, "Voilà", in La terrasse, 10 avril, 2008.

8- JOLY, Genviève, "Rythme", in Poétique du drame et contemporain, n. 22, 2001.

9- KROL, Aurore, "Les âmes assassinées de Minyana", in Les Trois Coups, 13 février, 2012.

10- MINYANA, Philippe, "La parole irrépressible", dans Mardi Du Théâtre de France- culture, 25 février, 1992. 11 , "La Tour de Montaigne", in Les cahiers de Prospero, n. 3, décembre, 1994. $12-$ , "La lettre d'Atlantique", citée dans

Chambres/inventaires, dossier de Marie- Pia Bureau, CNDP, 1999.

(Dynamique de l'expression silencieuse ...) Dr. Alaaedin Baheidin Alaaedin. 
$13-$ "Ceux-là qui font écrire. Notice", in La revue Spectre, n. 2, 1999. 14 , "L'écrit", in Communication, Thêâtre d'aujourd'hui, Paris, Seuil, n 83, 2008, pp. 115-122.

15- RYNGAERT, Jean-Pierre, "Le personnage théâtral contemporain: symptôme d'un nouvel "ordre" dramatique", in L'Annuaire théâtrale, (43- 44), 103- 112, 2008.

16- RUZET, Séverine, "Dialogue face à la mort", in Dialoguer vol II, Revue Etudes théâtrales, 33/2005. 17- ZANDE, Nicole, "Entretien avec Nathalie Sarraute à propos du Mensonge et du Silence", in Le Monde, 18 janvier, 1967.

(Dynamique de l'expression silencieuse ...) Dr. Alaaedin Baheidin Alaaedin. 
"حركية التعبير الصامت في العمل الارامي لفيليب مينيانا"

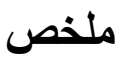

هذه الدراسة تتصب على الكتابة الدرامية للكاتب فيليب مينيانا. فيمكن القول أن

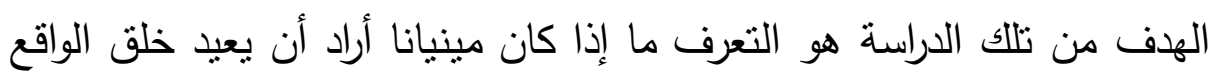
العادي لمسرحيات المسرح الكلاسيكي مستخما الحديث العام الذي يمكن ان يلامس شعور الجمهور من القراءمحاولا ان يعيد نلك العامية اليومية عن طريق

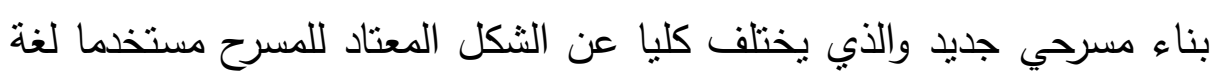
شاعرية معدة بشكل جيد. فالدراسة سوف تركز بشكل أساسي على موضوع

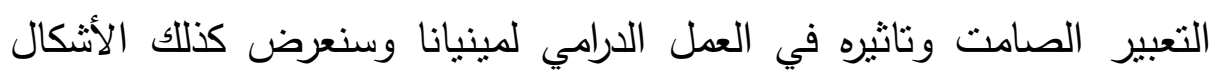
والنماذج المختلفة للتعبير الصامت المستخدمة بواسطة مينيانا في المسرحيات محل الدراسة في هذا البحث وهي: "بيت الموتى" و "مسرحيات" و"العودة" لأن الصمت في تلك المسرحيات يمثل طريقة لغوية يمكنها أن تعطي بعدا شاعريا لمسرحه.

كلمات مفتاحية: حركية -التعبير الصامت - العمل الارامي - فيليب مينيانا

(Dynamique de l'expression silencieuse ...) Dr. Alaaedin Baheidin Alaaedin. 